\title{
La enfermedad de Chagas como un rezago social en salud
}

\author{
Chagas's disease as a social remainder in health care
}

\section{Silvia María Guadalupe Pérez, ${ }^{\mathrm{I}}$ Edelsys Hernández Meléndrez, ${ }^{\text {II }}$ Aida Rodríguez Cabrera ${ }^{\mathrm{III}}$}

IDoctora en Medicina. Máster en Salud Poblacional con área de concentración en Epidemiología. Escuela Nacional de Salud Pública. La Habana, Cuba.

II Licenciada en Psicología. Doctor en Ciencias Psicológicas. Profesor Titular. Escuela Nacional de Salud Pública. La Habana, Cuba.

IIILicenciada en Economía. Doctor en Ciencias Económicas. Profesor Titular. Escuela Nacional de Salud Pública. La Habana, Cuba.

\section{RESUMEN}

La enfermedad de Chagas constituye un problema de salud característico de zonas rurales y suburbanas, muy ligado a la pobreza. Su solución requiere de políticas y estrategias que erradiquen su presencia en los países afectados. En el Estado de Tabasco, México, aún persiste esta enfermedad, lo que constituye un estigma social. Es por ello que se realizó una valoración de sus factores determinantes, su trascendencia como rezago social y de salud y las implicaciones bioéticas que surgen de la actuación de los servicios de salud hacia la población que la padece. El presente trabajo es resultado de una revisión documental donde se muestra cómo esa enfermedad necesita urgentemente un abordaje desde el paradigma médicosocial, que propicie su análisis desde la perspectiva multi e interdisciplinaria para desarrollar su prevención integral, desde el diagnóstico hasta la rehabilitación, en las dimensiones individual y colectiva, con énfasis en la promoción y protección de la población. Se indica la necesidad de rediseñar las organizaciones de salud, hacerlas más funcionales, eficientes y eficaces de forma tal que armonicen con los principios éticos, los valores y los intereses de la población, donde estén presentes el compromiso y la acción para erradicar la enfermedad en el Estado de Tabasco.

Palabras clave: Enfermedad de Chagas, paradigmas de salud, ética de salud. 


\begin{abstract}
Chagas' disease is a health problem inherent to the rural and suburban areas and very much linked to poverty. The implementation of policies and strategies that eradicate its presence in the affected countries will solve this problem. In Tabasco, Mexico, this disease still persists as a social stigma. This is the reason why the determining factors of the disease, its significance as a social remainder in health and the bioethical implications stemming from the performance of the health services in relation to the affected population were all assessed in this paper. This paper resulted from a documentary review which showed how this disease needs to be urgently addressed from a social and medical paradigm. The latter will allow analyzing the disease from a multi-and interdisciplinary perspective in order to develop comprehensive prevention ranging from diagnosis to rehabilitation, both individually and collectively, with emphasis on the specific protection of the population. Also, the need of redesigning the health organizations to turn them into more functional, efficient and effective bodies were emphasized, so that they take into account the ethical principles, the values and the interests of the population and reflect the commitment and the actions to eradicate this disease in Tabasco.
\end{abstract}

Key words: Chagas' disease, health paradigm, health ethics.

\title{
INTRODUCCIÓN
}

En la actualidad la enfermedad de Chagas en Tabasco se ha convertido en un problema de salud en la población rural de escasos recursos y ocasionalmente en la proveniente de áreas urbanas y periurbanas en las que sus habitantes viven en condiciones precarias.

Los últimos casos de mortalidad diagnosticados con esta enfermedad y que han sido detectados por hallazgos en el segundo nivel de atención, han demostrado que las estrategias del programa nacional y local realizadas para la vigilancia, prevención y control de la enfermedad de Chagas, no se ejecutan de manera eficaz, por lo que existe una deficiente detección de los casos. Ello resulta en un subregistro u omisión de enfermos, poca actividad e intervención activa en la búsqueda del vector y su control en áreas de riesgo, lo que lleva a realizar un breve análisis desde el enfoque del paradigma biologicista, transitando por el multicausal, para concluir con el paradigma médico-social y los beneficios en la salud de la población.

A partir de estas precisiones, el propósito de este artículo es, mediante una revisión documental, realizar una valoración crítica de los factores determinantes de la enfermedad de Chagas, su trascendencia como rezago social de salud y las implicaciones bioéticas que surgen de la actuación de los servicios de salud hacia la población que padece esta enfermedad, con énfasis en el Estado de Tabasco, México.

Un aspecto importante lo constituyen las consecuencias de la enfermedad de Chagas en la población y la magnitud de los problemas de salud que se presentarán en los próximos años en caso de no reorientarse las políticas para el fortalecimiento 
de las estrategias en su más amplia expresión, concebidas para mitigar el daño y los efectos en las futuras generaciones en el Estado de Tabasco. También cabe destacar el impacto inmediato, a mediano y largo plazo de la enfermedad en el deterioro del estado de salud de la población, lo que incrementará el gasto en salud por el peso de esta afección.

Un aspecto poco tratado se refiere a los compromisos y responsabilidades de cumplir los principios éticos por parte de los servicios de salud con la población que padece la enfermedad de Chagas, los que lamentablemente en ocasiones se encuentran ausentes de diferentes instituciones de salud.

Dado lo complejo y delicado del tratamiento de los cuestionamiento éticos en este problema de salud, su abordaje representa un reto, más aún cuando existe en el Estado un programa para la solución de este problema de salud, el que es incompleto y funciona con múltiples deficiencias.

Una visión acerca de la situación señalada puede valorarse mediante el análisis de los antecedentes, políticas de salud vigentes, población y superestructura ideológica, nivel de los recursos científicos-técnicos disponibles para su abordaje, financiamiento, determinación del personal de investigación acerca de la enfermedad de Chagas en Tabasco, México, que no dista mucho de la situación existente en otras regiones y países del mundo y en particular de América Latina, lo que según Joao Carlos Pintos, 1988: "la enfermedad es una mancha que categoriza, que define pobreza, que marca las dificultades político-administrativa de los sistemas de salud de los países latinoamericanos." ${ }^{1}$

\section{FACTORES DETERMINANTES DE SALUD EN LA ENFERMEDAD DE CHAGAS, COMO REZAGO SOCIAL DESDE LOS PARADIGMAS DE LA SALUD PÚBLICA}

Los determinantes de la salud son las condiciones que contribuyen al nacimiento y mejoramiento del estado de salud de los seres humanos, y es el nombre asignado a los factores que se cree influyen en la salud de una población. Un elemento importante de esta definición es la noción de que los mismos no actúan aisladamente sino que las interacciones complejas entre estos factores tienen una repercusión aún más profunda en la salud. ${ }^{1,2}$ En este sentido, una vez más se cumple el principio de determinación donde todo es condicionado según leyes.

En este contexto los indicadores determinantes de la salud pueden dividirse en categorías: comportamientos y estilo de vida, influencias dentro de las comunidades que pueden reforzar la salud o dañarla, las condiciones de vida y de trabajo, el acceso a los servicios sanitarios y las condiciones generales de tipo socioeconómico, cultural y medioambiental existentes. La actuación en el ámbito de los principales factores determinantes de la salud tiene un gran potencial para reducir los efectos negativos de las enfermedades y promover la salud de la población. ${ }^{3,4}$ El desequilibrio o la ausencia de estos componentes de salud en la enfermedad de Chagas, son detonantes para el incremento en la incidencia de los casos en cualquier población y el número de prevalentes sin tratamientos.

La enfermedad de Chagas es la zoonosis parasitaria más importante en América Latina, actualmente ocupa el cuarto lugar en importancia como causa de discapacidad, después de las afecciones respiratorias, las diarreas y el VIH/sida. Se encuentra entre las seis enfermedades prioritarias para la investigación, 
tratamiento y control de enfermedades tropicales según la OMS. Después del paludismo, es la enfermedad más grave e importante y representa un problema de salud de alcance en 17 países latinoamericanos, con un total estimado de 100 millones de personas expuestas a la enfermedad y de 16 a 18 millones infectadas. ${ }^{5}$

Es una enfermedad parasitaria causada por el protozoario flagelado Tripanosoma cruzi. Su transmisión al ser humano y algunos animales es a través de los insectos triatominaes (chinche hocicona, vinchuca, chick, talaje, chipo, barbeiros) en el momento que perforan la piel para succionar la sangre que los alimenta (alimentación nocturna), sin embargo, no se inocula directamente por las estructuras bucales del insecto sino que se deposita pasivamente en la piel a través de las heces y penetra al organismo por la herida que causa la picadura. ${ }^{6}$

Esta enfermedad se relaciona con el desarrollo económico y social, por lo que persistirá mientras existan los factores de riesgos más importantes para la domiciliación de los triatómicos, tales como vivienda de mala calidad, la migración frecuente de personas y la rápida urbanización. ${ }^{5,6} \mathrm{~A}$ estos se agregan los bajos ingresos, que no permiten mejorar las condiciones de vida y vivienda de la población. Esto hace que la mayor incidencia de la enfermedad se concentre en los países pobres.

\section{La enfermedad de Chagas desde los paradigmas biologicistas y multicausal}

Se trata de una enfermedad dentro del grupo de las infecciosas transmisibles por vector. Esto tal vez obedece más a la conducta biologicista de los profesionales de la salud, que al poco o nulo impacto que tiene la presencia de un caso en el cual las manifestaciones agudas se presentan por regla general de forma aleve. Se ha caído en el grave error de omitirlo de la agenda de salud, dado por el comportamiento de la historia natural de la enfermedad, en la que las personas que la sufren no presentan en forma inmediata complicaciones convincentes de la magnitud del daño que tendrán. Estos efectos se ven con mayor frecuencia en aquellas poblaciones pobres, rezagadas en salud y educación, lo que conduce al cuestionamiento de si la enfermedad de Chagas es un rezago social de salud, y si efectivamente los paradigmas biologicistas y multicausal, se encuentran representados en algún momento en el espectro de la enfermedad.

Si se analizan los eventos por separados, es decir, la identificación de un caso crónico en un segundo nivel, se tendría una concepción puramente biologicista al tratar solo a este paciente sin realizar estrategias de búsqueda activa y control del vector, por otro lado, si se valora el hecho de que se detecte un caso agudo, es decir, en la primoinfección en una unidad de salud de alguna comunidad, se reporte y se realicen estrategias integrales, se estarán realizando a partir de soluciones de la red problemática desde el paradigma multicausal-ecológico, que se enfoca hacia el paradigma médico-social.

La enfermedad de Chagas es un claro ejemplo de cómo la ausencia o limitación de los determinantes de la atención de la salud, repercuten en la calidad de vida de las poblaciones humanas en condiciones de riesgo, generado por la presencia del vector y la falta de estrategias para su control. Si continua el estudio de lo individual sobre lo colectivo, y no se trabaja de manera preventiva para la enseñanza del autocuidado de la salud, en consecuencia, se tendrá una gran cantidad de personas expuestas al contagio de la enfermedad y por ende, la producción de servicios curativos más que preventivos, que en cierta forma pueden llegar a ser costosos, elitistas y tecnificados. 
Ante la variabilidad de sus manifestaciones y sus características epidemiológicas entre una y otra zona endémica, así como los efectos de la enfermedad sobre las poblaciones pobres afectadas, se han realizado movimientos a favor del reconocimiento de la gravedad que representa. Ello ha derivado en iniciativas y políticas internacionales para el abordaje de este problema de salud, que pretenden romper con el paradigma biologicista, para dar paso al paradigma multicausal o ecológico como un modelo consolidado en 1960, acotándose a la definición de salud de la OMS, pero estancándose y fluctuando entre un paradigma y otro, durante su transición al paradigma médico-social.

Las estrategias que se han impulsado a través de programas que cuentan con resultados exitosos, cuyas acciones primordialmente están enfocadas a la eliminación del triatominae, el tamizaje de la sangre de transfusión y el apoyo terapéutico para los casos infectados con Tripanosoma cruzi, no hacen otra cosa que demostrar que el enfoque preventivo y el paradigma médico-social aún se encuentran ausentes y sin influencia en las políticas de salud.

A lo anterior se debe agregar que si se desea ver con optimismo el modelo que actualmente busca empoderarse para la solución de los problemas de salud actuales provocados por la enfermedad de Chagas y sus complicaciones, hay que reconocer algunas de sus bondades y cómo a pesar de su arraigo al flexnerismo, existe en este modelo un concepto menos rígido de las especialidades. En el mismo se acepta que los factores sociales suelen funcionar como agentes causales de enfermedad, promueven la prevención y promoción para la erradicación de la enfermedad y se realizan prácticas de campo cuyo enfoque es la captura e identificación del vector transmisor de la enfermedad. Sin embargo, el componente social en cuanto a la responsabilidad y proyección social de los profesionales y demás personas involucradas, prácticamente se dan en forma aislada, lo que traduce en una población a la que le falta mucho que aprender. En México y en particular en el estado de Tabasco el paradigma médico- social se presenta aislado y sin fuerza.

\section{El paradigma médico-social: una solución para la enfermedad de Chagas}

El paradigma médico-social es visto por la comunidad internacional como una de las mejores soluciones a los grandes problemas de salud que afectan el planeta, por ser su objeto de estudio los problemas de salud y enfermedad visto como procesos, en la enfermedad de Chagas esto es fundamental. El mismo es considerado como una posible solución para la prevención y control de esta enfermedad, dado que el proceso de salud-enfermedad se desarrolla en una comunidad, dentro de una colectividad, determinado por el modo específico en que se da el proceso biológico, de reproducción y la unidad interna de sus redes sociales para la solución ce los problemas de salud que acarrea la enfermedad.

En el estado de Tabasco hay procesos de producción que dan origen a los perfiles epidemiológicos de clase, vinculados con el estatus económico de las poblaciones y asociados, frecuentemente, a enfermedades que están presentes a lo largo de su vida, como es el caso de la enfermedad de Chagas, que existe en zonas donde viven o subsisten poblaciones en condiciones de extrema pobreza y precarias condiciones de salud.

Sin embargo, el problema consiste en que las relaciones entre el Estado, las condiciones de salud de las poblaciones pobres expuestas a la enfermedad de Chagas y las políticas de salud existentes, no permiten dar adecuada y rápida 
respuesta social organizada a las posibles demandas que genera la enfermedad y con ello, a las implicaciones bioéticas desde el punto de vista social.

La enfermedad de Chagas necesita urgentemente un abordaje desde el paradigma médico-social y propiciar su análisis multi e interdisciplinario, con el fin de desarrollar la prevención integral, desde el diagnóstico hasta la rehabilitación en las dimensiones individual y colectiva con énfasis en la promoción y la protección específica. Por último, se requiere de la realización de investigaciones basadas en la realidad social, el desarrollo e impulso de programas de investigación

multidisciplinarios e interinstitucionales en los que se haga el estudio integral de la enfermedad de Chagas y propicien a la vez, el desarrollo de tecnologías apropiadas a la realidad social.

Todo esto sería ideal, sin embargo la dimensión real, es que en los servicios de salud se siguen aplicando, en gran medida, la teoría unicausal y se continúa privilegiando los procedimientos curativos rehabilitatorios, así como la supremacía de las especialidades (Flexnerismo), por lo que persisten hasta la fecha y no se promueve la necesidad de relacionar los contenidos de las áreas biológica, clínica y social con el proceso salud-enfermedad del individuo. EL análisis de la situación de salud tiene limitaciones en la práctica, solo se realizan levantamientos de índices epidemiológicos, sin articulación con la clínica integral y no se consideran todos los factores que intervienen en el proceso.

\section{IMPLICACIONES BIOÉTICAS DE LOS SERVICIOS DE SALUD HACIA LA POBLACIÓN Y SU RELACIÓN CON LA ENFERMEDAD DE CHAGAS}

\section{La enfermedad de Chagas, un problema de salud de exclusión por omisión}

La omisión, desde el enfoque social, es vista como una modalidad delictiva en la cual se incurre al adoptar una actitud pasiva ante los preceptos positivos de leyes o normas denominado como delitos de simple omisión. Mientras que el simple no hacer, se produzca o no, un resultado constituye autónomamente la infracción en los delitos de omisión. Es aquí donde se introduce el término omisión de deberes, en la que la omisión es vista como un delito de abuso de autoridad, referida a la conducta de los funcionarios públicos que dolosamente dejan de cumplir o retardan el cumplimiento de sus obligaciones propias, desatendiendo los requerimientos recibidos al efecto. ${ }^{1}$

En la enfermedad de Chagas, la exclusión del grupo de enfermedades infecciosas cuyo control y vigilancia se encuentran legislados y normados, no se realiza por encontrarse en n grupo denominado de prioridad II, sin embargo, aún en este grupo es mencionada, pero finalmente se le termina excluyendo. Por ello es necesario entender hasta dónde llega el alcance de este concepto y en este sentido la exclusión es más que el mantenimiento de la homogeneidad o integridad de un grupo eliminando a personas cuyas características de conducta o calidad se consideran como una amenaza para su solidaridad. Este término ubicado en las políticas, muchas veces se observa por extensión, y la política de exclusión comprende la expulsión de indeseables por iguales motivos. En los servicios de salud, la enfermedad de Chagas es vista como una enfermedad indeseable, cuyo control y vigilancia no es un problema, por lo que se termina realizando una exclusión por omisión. 
Esta enfermedad afecta en particular a individuos que habitan en zonas rurales y suburbanas y está ligada a la pobreza. Constituye una enfermedad social, por lo que la habitación donde pueden estar los enfermos desempeña un papel decisivo en la transmisión, ya que la vivienda campesina, precaria, hecha de materiales diversos, como el adobe, con techo de materia vegetal y con piso de tierra, se convierte rápidamente en un criadero de chinches, que ya no necesitan salir para buscar su alimento. Estudios realizados anteriormente han demostrado que algunas especies muestran una tendencia a ocupar los hábitats más estables a su alcance, entre los que se encuentran el ambiente doméstico y peridoméstico. Esto quiere decir que existen especies que anteriormente se encontraban únicamente en forma silvestre, pero que se han adaptado al invadir paulatinamente las viviendas y sus alrededores. ${ }^{6,7}$ (Ransey-Willoquet J. Estratificación de municipios y comunidades de riesgo para la transmisión vectorial de la Enfermedad de Chagas en un estado. Instituto Nacional de Salud Pública. Documento inédito preliminar. Cuernavaca, Morelos 2002).

Según cálculos realizados en 1981 por la OPS y la OMS, Latinoamérica tenía 24 millones de infectados y 65 millones en riesgo. En México, Schofield en 1985 calculó 3 millones 800 mil infectados basándose en los datos de Goldsmith y otros, con casi un millón más de los calculados por Velasco Castrejón para el mismo año, y con cifras superiores a lo observado en la encuesta serológica nacional, en la cual los estados que tuvieron mayor prevalencia de infectados no sobrepasaron el $1 \%$, por lo que se puede calcular en varios cientos de miles los mexicanos infectados. ${ }^{2,8}$ Sin embargo, debido a que la enfermedad se distribuye en focos, es posible que muchos casos no se hayan investigados durante la encuesta seroepidemiológica y sean en realidad muchos más los afectados para los períodos señalados.

A partir de 1993 la OMS la consideró como la enfermedad parasitaria más grave en América y es parte de la lista de las 14 enfermedades descuidadas o "negligidas" según la OMS. Recientemente esta organización reconoce a la enfermedad de Chagas como un problema de salud global dados los movimientos migratorios. Países no endémicos como España ya han comenzado a implementar mecanismos de detección de la enfermedad en donadores inmigrantes, ya que cada año recibe más inmigrantes latinoamericanos. En México, durante el período 2001 a 2006, se notificaron 1616 personas infectadas, de las cuales el 12,2\% eran menores de 14 años, el grupo de 15 a 44 años representó el 58, $7 \%$ de los casos. ${ }^{9}$ En 2005 se registraron estimaciones para México que se muestran en la tabla. 
Tabla. Estimaciones de la enfermedad de Chagas. México, 2005

\begin{tabular}{|l|c|}
\hline Datos & Año 2005 \\
\hline Población & 107029000 \\
\hline No. de infectados & 1100000 \\
\hline Nuevos casos anuales de transmisión vectorial & 7700 \\
\hline Casos anuales de Chagas congénito & 1,1 \\
\hline Mujeres (+) 15 y 44 años & 243 \\
\hline Tasa de incidencia ( $\times 100$ habitantes) & 1028 \\
\hline Tasa de prevalencia ( $\times 100$ habitantes) & 0007 \\
\hline Incidencia de Chagas congénito (× 100 nacimientos) & 29500000 \\
\hline Población expuesta en zonas endémicas & 99143 \\
\hline Miocardiopatías & 0,60 \\
\hline Prevalencia en bancos de sangre & \\
\hline
\end{tabular}

Nota: estimación cuantitativa de la enfermedad de Chagas en Las Américas. $\mathrm{OPS} / \mathrm{HDM} / \mathrm{CD} / 425-06$.

Diciembre 2006. Montevideo, Uruguay. p. 28.

En el 2008, la enfermedad de Chagas es reconocida por la OMS/OPS, dentro del grupo de enfermedades tropicales denominadas como desatendidas. En la actualidad esta enfermedad no se limita a las latitudes tropicales y prácticamente afecta a más de mil millones de personas en el mundo. Casi el $100 \%$ de la carga de la enfermedad se limita a al región de Las Américas, pero, como ya se dijo, existen casos importados en Europa y otros lugares. El Plan Global de la OMS para la lucha contra las enfermedades tropicales desatendidas del período 2008-2015 lanzó una nueva iniciativa para eliminar a la enfermedad de Chagas como problema de salud pública. ${ }^{10}$

Las cifras han variado hacia la disminución de anteriores estimaciones, pero ello lo explican ajustes metodológicos de los cálculos y mejores encuestas, así como también éxitos de la prevención y control en múltiples países y regiones donde se llevan a cabo programas de control antivectorial.

\section{Implicaciones bioéticas de los servicios de salud en la enfermedad de Chagas. Un problema de salud previsible}

La enfermedad de Chagas es una enfermedad altamente previsible ya que responde favorablemente a todas las medidas de control dirigidas al vector transmisor de la enfermedad. Sin embargo, cuando las acciones para la atención de este problema están ausentes, la enfermedad se desarrolla de forma acelerada por la poca o nula respuesta de la institución a esta prioridad de salud de las poblaciones enfermas, que se llegan a considerar de alto riesgo. La falta de una respuesta social organizada y oportuna de los servicios de salud, en relación con la enfermedad de Chagas, no logrará disminuir los rezagos en aquellas poblaciones en las que la brecha de salud es mayor que en otras, y en las cuales existen graves problemas relacionados con al accesibilidad a estos servicios, y a esto se agregan la falla en la toma de decisiones y el poco o nulo compromiso con la población por parte de las autoridades así como las formas en que llegan hasta ellos los servicios vitales y 
necesarios para conservar la armonía, el equilibro y la estabilidad de la salud social. ${ }^{11}$

Ante este panorama y con el afán de mantener la equidad y la justicia social, el gobierno mexicano ha desarrollado una serie de políticas, leyes, normas y reglamentos de salud vigentes que garantizan la protección contra los riesgos sanitarios de los individuos, respetando así sus garantías individuales y salvaguardando por ley su salud como un patrimonio del estado ante las enfermedades previsibles por naturaleza.

Es aquí donde se analiza el término "previsible", que se refiere a los elementos característicos de la culpa, que consiste en la posibilidad de prever los resultados dañosos de la acción no previstos efectivamente en el caso de que se trate, constituyendo la negligencia. Por ello, la previsibilidad significa la concepción sanitarista de la visión de un futuro mejor posible y donde la vivacidad de tal imaginación puede actuar como una amenaza o como una promesa ejerciendo así un control personal o social. ${ }^{1}$

A continuación se realizan algunas consideraciones jurídicas y normativas propias del programa actual, complementado con aquellas dirigidas a la población beneficiada.

En México, la Constitución en su artículo 4, expresa que la salud es un bien universal y que todo mexicano tiene derecho a la protección de su salud. La Ley General de Salud Mexicana en su Título Segundo, capítulo 1 de su artículo 5to. dice: "tiene por objeto dar cumplimiento al derecho de la protección de la salud", compromiso que se ratifica en el capítulo II y $\mathrm{V}$ en sus artículos 58 y $59 .{ }^{12}$

Es importante mencionar que el Título VIII para la vigilancia epidemiológica, prevención y control de enfermedades, integra a la tripanosomiasis americana (enfermedad de Chagas), como una más de importancia en México, para su estricta vigilancia y control en apego a la normatividad, sin embargo el cumplimiento a este mandato es parcial, por lo que la falta de previsibilidad planificada en la enfermedad de Chagas, por las autoridades sanitarias compromete y cuestiona seriamente su deber, responsabilidad, justicia sanitaria y calidad de la atención, así como su efectividad y eficiencia. ${ }^{10}$

\section{CARACTERÍSTICAS DEL SISTEMA SOCIAL MEXICANO Y SU VÍNCULO CON LA ENFERMEDAD DE CHAGAS}

El Programa Nacional de Salud 2001-2006, 2007-2012, en sus estrategias destaca el vínculo de la salud con el desarrollo económico y social, la reducción de los rezagos en salud que afectan a los pobres, el enfrentamiento a los problemas emergentes y reemergentes para alcanzar la equidad y la justicia social y dé respuesta social organizada a enfermedades que son un atraso en materia de salud y de alto impacto económico y social para el país. ${ }^{13}$

En este sentido, la respuesta social organizada ha sido rebasada ante la presencia de la enfermedad de Chagas, por lo que estas reflexiones se apoyarán firmemente en las premisas antes mencionadas, si se tiene como base la voluntad política para la atención de este problema de salud. 
En México el Programa de Acción específico 2007-2012, "Otras Enfermedades Transmitidas por Vector" es uno de los muchos programas prioritarios, que buscan resolver y dar respuesta a problemas y demandas de salud generadas por las enfermedades transmisibles por vectores y por diversos agentes etiológicos, que generalmente se asocian o se ven vinculadas a las malas condiciones de vida generadas por la pobreza. ${ }^{14}$

Este programa tiene un enfoque antivectorial aunado al mejoramiento de las condiciones de vida de las poblaciones expuestas, y se ha realizado basado en el proyecto de Norma Oficial Mexicana PROY-NOM-032-SSA2-2000, ${ }^{14}$ y de último, de la Norma Oficial Mexicana de Emergencia NOM-EM-003-SSA2-2003, para la vigilancia epidemiológica, prevención y control de enfermedades transmitidas por vector. ${ }^{15}$ En dicha norma se plantea el abordaje de las enfermedades transmisibles por vector, de una manera integral holística, donde se considera para cada una en particular los siguientes componentes estratégicos: Saneamiento Básico y Mejoramiento de Vivienda, Eliminación de Larvas y Criaderos (que se ha modificado en el bloque de eliminación de larvas y criaderos, colocando ninfas y criaderos), Disminución de la Cantidad de los Vectores, Diagnóstico Oportuno y, Tratamiento o Manejo Hospitalario Adecuado. En la (figura).

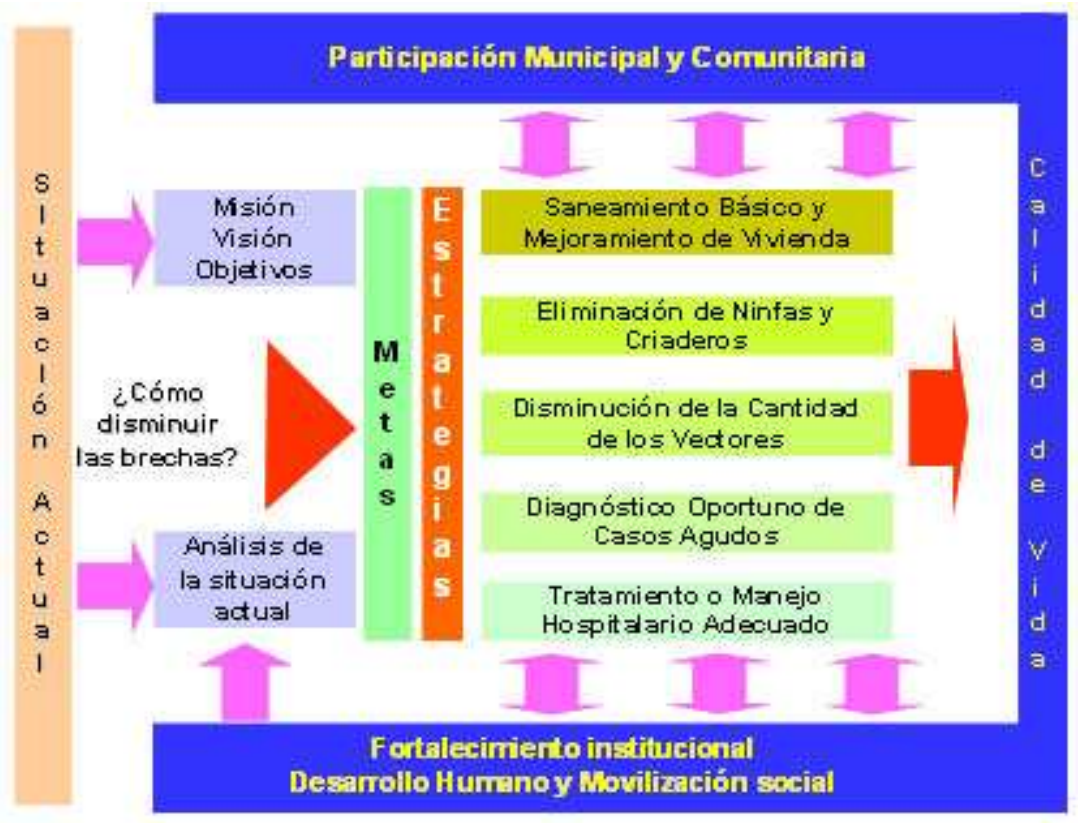

Fig. Componentes estratégicos del programa de acción.

El cumplimiento de estos componentes, generalmente se asocia con el logro exitoso de las metas del control del vector, y por consiguiente con la disminución de la morbilidad y mortalidad, situación que no se encuentra desarrollada en Tabasco.

En México, así como en los países endémicos a la enfermedad de Chagas, el programa está dirigido particularmente para el control del tríatoma, el cual considera los siguientes aspectos: datos básicos de las áreas de riesgo epidemiológico y las diversas actividades derivadas de los componentes estratégicos, que se abordan de acuerdo a: saneamiento básico y mejoramiento de vivienda, que se realizan a través de actividades dirigidas al ordenamiento del medio; la eliminación de las crías y criaderos y disminución de la cantidad de los vectores, se ejecuta por medio de los estudios entomológicos y la aplicación del rociado domiciliario; el diagnóstico oportuno, que utiliza la pesquisa domiciliaria de 
casos y los casos diagnosticados oportunamente por el primer nivel de atención y por último, el tratamiento o manejo hospitalario adecuado, con el auxilio de los casos a tratar y los registrados. Sin embargo, no se cumple con estas premisas.

\section{Factibilidad de recursos para lograr la justicia sanitaria}

En este sentido, es fundamental la factibilidad que pueda existir en cuanto a los recursos para la búsqueda y logro de la "justicia sanitaria", viéndola desde la óptica de una necesidad para conseguir el bien universal que representa la salud y su importancia como bien estratégico para el desarrollo de los pueblos, vinculándose a estos el carácter de sus necesidades y cómo se vincula con otros bienes básicos. Sin embargo, la restricción y limitantes de este modelo radica en:

- la escasez de recursos,

- las características profundamente inequitativas de la sociedad mexicana.

Esta situación permite avanzar en el sentido de analizar las nuevas orientaciones de las políticas que priorizan la libertad y la responsabilidad individual (principio de autonomía), por sobre el principio de justicia, que reducen el protagonismo del estado a su mínima expresión y por lo mismo incrementan las inequidades.

A lo anterior, se agrega el alto costo que demanda hoy la atención de la salud y es ante esta situación, que se ve relegada y pierde vigencia lo que en el pasado sería "la salud como derecho y responsabilidad del estado", que queda hoy reducida a la atención de salud como una espectadora, mientras que la salud como bien de consumo 'bien de mercado' asumida de manera individual se propone hoy como un "conjunto básico de prestaciones."

Cuando se analizan los derechos y obligaciones que marcan las leyes, normativas y programas en vigor en materia de salud, así como las políticas de salud actuales en México y en el Estado de Tabasco, se considera necesario el análisis exhaustivo y reconocimiento de la enfermedad de Chagas, como un problema de salud serio a mediano y largo plazo. Enfrentarlo ahora, es ya un reto ético, y el fin es mitigar los daños presentes y futuros en la población.

El derecho a la salud en todo ser humano es un bien primario e inalienable, así como contar con respuestas institucionales efectivas en cuanto a su educación en materia de salud, el conocimiento de la detección de riesgos que puedan afectarle y sobre todo a la seguridad de tener servicios de salud que respondan organizadamente ante un problema como la enfermedad de Chagas, que propicien la posibilidad de detección oportuna, tratamiento y seguimiento de los casos hasta su curación (casos agudos), y en los casos crónicos tratamiento paliativo y acceso a los servicios de segundo y tercer nivel para su vigilancia y control, específicamente servicios de cardiología, gastroenterología y neurología, situación que presenta insuficiencias en el Estado de Tabasco.

\section{Financiamiento para la erradicación de la enfermedad de Chagas}

Cuando se habla de recursos financieros, el problema ético se hiperboliza, porque las necesidades de salud son infinitas y los recursos finitos y es aquí donde la 
institución se ve comprometida a cuestionar su propia ética institucional, colocándola en un dilema. Un dilema ético se presenta cuando al cumplir con un principio de la ética automáticamente se contraviene otro. ${ }^{16}$ Es conocer la existencia de los casos y hacer lo que se pueda con acciones aisladas según se presenten, aquí se contravienen los principios de equidad y beneficencia, que se acompañan generalmente con la falta de recursos humanos capacitados, carencia de medicamentos específicos por su costo elevado o su ausencia; en Latinoamérica, por falta de empresas farmacéuticas que lo desarrollen o por carencia de permisos para su compra/venta en el mercado mexicano, como es el caso del Benzonidazol y el Nifurtimox. También por estudios de gabinete inaccesibles para la mayoría, y para los casos complicados, dificultades para realizarse intervenciones de especialidad de alto costo, como la adquisición de un marcapaso y otros que pueda requerir el paciente.

El programa de prevención, control y vigilancia para la enfermedad de Chagas, tal como existe hoy en México, no cuenta con financiamiento o clave presupuestal asignada, y entonces la pregunta es ¿qué pasa con los enfermos detectados por hallazgo en donantes de sangre?, ¿el banco de sangre los canalizó hacia algún servicio médico para su manejo y vigilancia?, y en el peor de los casos ¿cuántas mujeres expuestas se infectan y tendrán hijos seropositivos al parásito, que sufrirán la enfermedad?, ¿cuántos niños de los que ingresan a los hospitales estarán ahora en las últimas fases de una cardiopatía chagásica?, y una pregunta que golpea es ¿cuántos niños se mueren ahora, porque el medicamento faltó? ¿Es esto importante, como para que se considere una propuesta formal de asignación presupuestal a este programa ante el reconocimiento de su existencia e impacto en la salud de la población?, ¿es acaso posible considerar en la enfermedad de Chagas, la negación y la exclusión por omisión de su existencia y del verdadero problema actual que representa, como respuesta al problema ya existente? Existen otros cuestionamientos que implican a la ética y a la bioética, como son: asegurar la existencia de los tratamientos, proporcionar el mayor beneficio posible en cada caso específico, esto entre las muchas necesidades que existen en este programa y que hasta hoy prevalecen.

\section{Consecuencias de la enfermedad de Chagas en la población y los efectos en la sociedad y la economía}

El impacto social y económico de la enfermedad de Chagas es enorme, incluso ocurre que en algunos países, no se da trabajo a quien tiene una serología positiva. Por los numerosos factores involucrados, a los cuales se suman aquellos de poder político y económico, esta enfermedad pasa a constituirse no solo en una enfermedad de la pobreza, sino en un ejemplo de los mecanismos de ocultamiento y exclusión como forma de discriminación social y laboral. ${ }^{17}$

En las últimas dos décadas se han incrementado los conocimientos acerca de la enfermedad de Chagas, no solo mediante la investigación, sino entre las autoridades sanitarias, esto ha promovido el otorgamiento de beneficios financieros nacionales e internacionales para los países afectados, situación que refleja el compromiso político y técnico asumido por los países, lo que es de importancia para el desarrollo de programas de vigilancia a largo plazo. ${ }^{13}$

Todas estas iniciativas internacionales han contado con el respaldo de la OPS y las evaluaciones de las acciones han tenido resultados excepcionales en el control de la enfermedad de Chagas. ${ }^{13,18}$ 
Durante el año 2002, Ransey y otros, así como representantes de todos los Estados de México con casos de la enfermedad de Chagas, iniciaron un movimiento, con el fin de llamar la atención de las autoridades institucionales, sobre el rezago de esta enfermedad en el país y su incremento, debido a la ausencia de intervenciones en la mayoría de los Estados. En apoyo a esta iniciativa, estuvieron presentes expertos investigadores del Cono Sur, del Pacto Andino y de la Comunidad Europea, así como de la OPS y representantes de los servicios de salud mexicanos, quienes se comprometieron a realizar un análisis del problema, sin respuesta hasta ahora. ${ }^{19}$

En Tabasco, los servicios de salud realizan desde 1994 acciones aisladas dirigidas para el control del vector de la enfermedad de Chagas, sin embargo es en 1996, que se hace evidente la existencia de la enfermedad con el diagnóstico serológico de dos casos, realizado por el Instituto Nacional de Diagnóstico y Referencias Epidemiológicas de México (INDRE).

Actualmente el programa reconoce como áreas de exposición a 13 municipios de 17 que integran el estado de Tabasco, siendo estos Balancán, Cárdenas, Centro, Centla, Comalcalco, Cunduacán, Emiliano Zapata, Huimanguillo, Jalapa, Jonuta, Nacajuca, Paraíso y Tenosique, quienes han reportado 45 casos positivos al parásito por laboratorio, hecho que exige realizar un diagnóstico de la situación actual del problema para fortalecer las acciones dirigidas al abordaje del control del vector, detección de casos y vigilancia de la enfermedad de Chagas.

En el Estado de Tabasco las actividades para el control vectorial son eventuales y parciales, y solo se realizan ante la presencia de algún caso crónico. Estas acciones se basan en el estudio entomológico y rociado domiciliario como el programa lo específica, sin embargo, la captura del vector es poco realizada y los productos químicos utilizados son específicos de otros programas de vectores. En cuanto al tratamiento, los servicios de salud no cuentan con el abasto de medicamentos específicos necesarios para tratar los casos agudos, por lo que se requiere solicitar la ayuda a otros países e incluso de la OPS, sin embargo la mayoría de las veces, el medicamento llega cuando ya no se puede hacer nada por el paciente. Estas circunstancias, muestran un programa debilitado que requiere ser fortalecido, además de lo injusto que es padecer una enfermedad o morir a consecuencia de una enfermedad altamente previsible.

\section{Población y superestructura ideológica}

La intervención para el abordaje y control de la enfermedad de Chagas en las comunidades deberá ser con el máximo respeto y cuidado a la integridad, condiciones de vida, ideologías, cultura, sus tradiciones y sus habitantes, manteniendo como objetivo primordial el respeto a la vida, que considere la salud de las personas como primera preocupación y no-discriminación para proporcionar atención. Para lograrlo se deberá seguir las recomendaciones que dicta la Ley General de Salud en materia de investigación, incluyendo cada uno de sus puntos.

En Tabasco, fundamentalmente en la zonas rurales, es preciso coadyuvar a la promoción de valores en la comunidad y establecer estrategias enfocadas a la educación ambiental y a la transmisión de conocimientos sobre la interdependencia de los procesos naturales y sociales, para generar cambios que perduren y aseguren un ambiente de calidad, en el que ni el hombre ni su entorno, sufran de alteraciones con el uso de los procedimientos de rociamiento con insecticidas. Una garantía de ello será mantener el área trabajada en vigilancia, que establezca comunicación permanente con las autoridades y la población, para atender de forma rápida y eficaz efectos negativos, después de la intervención. El compromiso 
será el apoyo al programa y a los tomadores de decisiones para su adecuado abordaje, pero aún más con la población que lo requiera.

Finalmente se puede concluir que la enfermedad de Chagas, constituye uno de los problemas prioritarios de salud pública debido a su amplia distribución, elevada prevalencia y a la disminución de la calidad y esperanza de vida de los que la padecen. La enfermedad sobre el hombre de hoy tiene una connotación e impacto no cuantificables aun para los servicios de salud, no solo en el peso económico, si no en el social. A esto se agrega la omisión de los deberes sanitarios para este problema previsible, que sin dudas constituye un signo de abandono social de la población del país donde está presente la enfermedad.

Debido a que la enfermedad es incurable, su control depende de su abordaje integral y multidisciplinario sobre aquellos hábitos que permiten mantener un contacto estrecho con el hombre o los animales domésticos, y que ocupa diversos refugios que ofrece la vivienda humana empobrecida, muy presente en el continente americano.

La solución de este problema en el ámbito de la salud solo puede mejorarse mediante un abordaje desde el paradigma médico-social. En el difícil camino de erradicar la enfermedad de Chagas es preciso potenciar la prevención y promoción en salud, lo que sin dudas coadyuvará a minimizar los problemas y dilemas éticos, esto por su puesto, insertado en un proyecto social que tenga como elemento básico la erradicación de la pobreza y la disminución de las desigualdades sociales en la sociedad mexicana.

\section{REFERENCIAS BIBLIOGRÁFICAS}

1. Pratt Fairchild H. Diccionario de Sociología. 2da ed. 1997. Fondo de Cultura Económica. LETRA O [sitio en Internet]. [citado 1 Dic 2009]. Disponible en: http://www.oei.es/salactsi/sanmartino.htm

2. Declaración de Yakarta sobre la Promoción de la Salud en el Siglo XXI. Promoción de la Salud. Yakarta: Dirección General de Salud Pública; 2004.

3. Sanmartino M. La enfermedad de Chagas desde un enfoque CTS. Centro de Enfermedad de Chagas. La Plata (Arg): Fundación INCALP. Laboratorio de Didáctica y Epistemología de las Ciencias, Univ. de Ginebra, Suiza [sitio en Internet]. [citado

1 Dic 2009]. Disponible en: http://www.oei.es/salactsi/sanmartino.htm

4. Informe de un Comité de Expertos de la OMS. Control de la Enfermedad de Chagas. [Serie de Informes Técnicos 811]. Ginebra: OMS; 1991.

5. Shofield CJ. Tripanosoma cruzi. The Vector-parasite Paradox. Mem Inst Oswaldo Cruz. 2000;95(4):535-44.

6. Carcavallo RU. Climatic factors related to Chagas disease transmission. Mem Inst Oswaldo Cruz. 1999;94(Suppl. 1):367-9.

7. Silva Aycaguer LC, Benavides Rodríguez A, Vidal Rodeiro CL. Análisis espacial de la mortalidad en áreas geográfica pequeñas. El enfoque bayesiano. Rev Cubana Salud Pública [serie en Internet]. 2003 [citado 1 Dic 2009];29(4). Disponible en: 
http://scielo.sld.cu/scielo.php?script=sci arttext\&pid=S086434662003000400004\&lng=es

8. OPS/HCP/HCT/192/01. Iniciativa DE Salud del Cono Sur (INCONOSUR) Xa. Reunión de la Comisión Intergubernamental para la eliminación de Triatoma infestans y la interrupción de la Tripanosomiasis Americana por transfusión. Montevideo (Uru): OPS/OMS; 2001.

9. Programa de acción específico 2007-2012. Otras enfermedades transmitidas por vector. Subsecretaría de prevención y promoción de la salud 2008. México, D.F.: Secretaría de salud; 2008.

10. Steven K. Chagas disease and Neglected Diseases: Challenging poverty and exclusion. La Enfermedad de Chagas. A la puerta de los 100 años del conocimiento de una endemia americana ancestral. OPS. OPS/CD/426-06. Fundación Mundo Sano. Monografía 7 [monografía en Internet]. 2007[citado 1 Dic 2009]. Disponible en: http://www.mundosano.org/documentos/unitarias/interior\%20libro.pdf

11. Dávalos HJ. La salud de la Globalización. Universidad Juárez Autónoma de Tabasco. Horizonte Sanitario. 2002,1(1).

12. Ley General de Salud. Agenda de Salud 2002. México, D.F.: Ediciones fiscales ISEF, S.A.; 2002.

13. Programa Nacional de Salud 2001-2006. México, D.F.: Secretaría de Salud; 2001.

14. Proyecto de Norma Oficial Mexicana PROY-032-SSA2-2000. Para la Vigilancia Epidemiológica, prevención y control de Enfermedades Transmitidas por Vector. Diario Oficial de la Federación. México, D.F.: Secretaria de Salud; 2001.

15. Norma Oficial Mexicana de Emergencia NOM-EM-003-SSA2-2003, Para la vigilancia epidemiológica, prevención y control de enfermedades transmitidas por vector. Diario Oficial de la Federación. México, D.F.: Secretaria de Salud; 2003.

16. Hernández E. Problemas éticos y psicológicos del trasplante de órganos. En aspectos psicológicos del trasplante de órganos. La Habana: Editorial Ciencias Médicas; 2008.

17. OPS/HCP/HCT/202/02. Cuarta Reunión de la Comisión Intergubernamental de la Iniciativa de Centroamérica y Belice para la Interrupción de la Transmisión Vectorial de la Enfermedad de Chagas por Rhodnius prolixus, Disminución de la infestación intradomiciliaria por Triatoma dimidiata y la eliminación de la Transmisión Transfusional del Tripanosoma cruzi. Montevideo (Pan): POS/OMS; 2001.

18. OPS/HCP/HCT/194/01. Grupo de Trabajo OPS para consulta en Planificación, Operativa, Estrategia y Evaluación de Etapas Avanzadas del Control Antivectorial en Enfermedades de Chagas. Montevideo: POS/OMS; 2001. 
Recibido: 8 de marzo de 2010.

Aprobado: 14 de agosto de 2010.

Silvia María Guadalupe Garrido Pérez. Dr. Jesús Menéndez Ruiz No. 8. Colonia Reforma, Villahermosa. CP 86080. Tabasco, México.

Teléf.: 52993 3154094. Correo electrónico: edelsys.hernandez@infomed.sld.cu; aidarc@infomed.sld.cu 